\title{
Industrias Creativas y Culturales: Estudio desde el Enfoque de la Gestión del Conocimiento
}

\author{
Carlos E. Blanco-Valbuena ${ }^{(1)}$, César A. Bernal-Torres ${ }^{(2)^{*}}$, Fernando Camachoo ${ }^{(3)}$ y Miguel Díaz-Olaya ${ }^{(4)}$ \\ (1) Universidad Santo Tomás de Bogotá, carrera 9 No 72-90, Bogotá, Colombia. \\ (e-mail: carlose.co2010@gmail.com) \\ (2) Universidad de La Sabana, Escuela Internacional de Ciencias Económicas y Administrativas, \\ Campus Universitario, Chía, Colombia (e-mail: cesar.bernal@unisabana.edu.co) \\ (3) Universidad de La Sabana, Facultad de ingeniería, Campus Universitario, Chía, Colombia. \\ (e-mail: fernando.camacho2@unisabana.edu.co) \\ (4) Carrera 97 \# 69-25, Bogotá, Colombia. (e-mail madodiaz@gmail.com)
}

* Autor a quien debe ser dirigida la correspondencia

Recibido Jul. 27, 2017; Aceptado Oct. 10, 2017; Versión final Nov. 27, 2017, Publicado Jun. 2018

\begin{abstract}
Resumen
Este artículo analiza el sector de las industrias creativas y culturales desde el enfoque de la gestión del conocimiento mediante análisis de datos agregados y desagregados. La información para este análisis se obtuvo mediante la aplicación de una encuesta a 110 directivos y empresarios de empresas del sector localizadas en Bogotá, Colombia. El análisis agregado de los datos indica que, en general, los encuestados reconocen la importancia de la gestión del conocimiento para construir ventaja competitiva en sus empresas pero que, al interior de éstas, se hace poca conversión de conocimiento tácito en explícito y poca transferencia de conocimiento entre las empresas. El análisis desagregado indica que la interiorización, comparada con la socialización, es el medio más usado por estas empresas para crear conocimiento. Estos resultados sirven de referente para que académicos y directivos empresariales y de organismos gubernamentales, interesados en las empresas de este sector, reflexionen y motiven acciones que permitan el diseño de estrategias de gestión del conocimiento tendientes a generar valor agregado y sostenible para estas empresas y sus diferentes grupos de interés.
\end{abstract}

Palabras clave: industrias creativas y culturales; gestión del conocimiento; conversión de conocimiento; creación de conocimiento, análisis agregado, análisis desagregado.

\section{Creative and Cultural Industries: Study from the Knowledge Management Approach}

\begin{abstract}
This article analyzes the creative and cultural industries sector from the perspective of knowledge management through the analysis of aggregate and disaggregated data. Information for this analysis was obtained through the application of a survey to 110 executives and entrepreneurs of companies in the sector located in Bogotá, Colombia. The aggregate analysis of the data indicates that the respondents recognize the importance of knowledge management to build competitive advantage in their companies, but that, within them, there are low levels of conversion of tacit knowledge into explicit and low transfer of knowledge among companies. The disaggregated analysis indicates that internalization, compared to socialization, is the most used means for these companies to create knowledge. These results serve as a reference for academic researchers and business executives, government agencies, and interested in companies in this sector, so they can reflect and motivate actions that allow the design of knowledge management strategies aimed to generating added and sustainable value and for these companies and their different stakeholders.
\end{abstract}

Keywords: creative and cultural industries; knowledge management; knowledge conversion; knowledge creation, aggregate analysis, disaggregated analysis. 


\section{INTRODUCCIÓN}

Las industrias creativas se están tornando cada vez más en importantes componentes de la sociedad postindustrial basada en el conocimiento. Estas industrias son responsables del más alto promedio de crecimiento y creación de empleo en los países desarrollados, son también vehículos de identidad cultural que juegan un papel importante en el fomento de la diversidad cultural (Dervojeda et al., 2013) y una apuesta por el valor añadido, la creatividad y la innovación. La creación de conocimiento y transferencia del mismo son las claves y fuente de la innovación, que a su vez es un factor relevante para la construcción de ventaja competitiva regional (Scherrer, 2008). Por otra parte, las industrias creativas y culturales han recibido mayor atención académica teórica y empírica en las últimas dos décadas (Cooke y Schwartz 2007; Andari et al., 2007, Hartley et al., 2012). Para los países nórdicos, el centro de Europa y los Estados Unidos, las industrias culturales son particularmente importantes fuentes de capacidades innovadoras e impulsoras del desarrollo económico y cultural regional. En estas regiones la creación y transferencia de conocimiento en las industrias culturales y entre éstas y otras industrias son importantes para el crecimiento económico, aunque los trabajadores en las industrias culturales abarca una fracción muy pequeña de la denominada clase creativa (Florida, 2002).

La contribución a la economía de Colombia por las industrias creativas y culturales es del $3.3 \%$ y del empleo el 5.8\% (Buitrago y Duque, 2013). De esta forma, Colombia ocupa el tercer puesto en Latinoamérica en número de empleados en este sector, después de México y Brasil. Por la importancia de estos datos y de los estudios realizados sobre el sector en el contexto mundial y en el de Colombia en particular, el objetivo de este artículo es analizar el perfil de la gestión y la creación de conocimiento en las industrias culturales y creativas mediante el procesamiento agregado y desagregado de datos y ofrecer un panorama de la denominada economía naranja y su relación con la economía basada en el conocimiento. Mediante la evidencia expuesta se pretende contribuir a la comprensión y el fortalecimiento de las industrias creativas desde el enfoque de la gestión del conocimiento. Para esto, la información se obtuvo mediante una entrevista en una escala likert a 110 directivos y empresarios de diferentes subsectores del sector, tales como: patrimonio y cultura, publicidad, diseño, diseño de software y 3D, multimedia, música, video juegos, artes y prensa. La clasificación de las empresas para este estudio se tomó de las referencias del Banco Interamericano de Desarrollo (BID).

La entrevista evaluó cuatro aspectos específicos de la gestión del conocimiento: las formas de conversión del conocimiento según el modelo $\mathrm{SECl}$, los medios de la generación de conocimiento, los responsables de la captura y adquisición de conocimiento y los beneficios de la gestión del conocimiento para la empresa. El análisis de los datos se realizó de forma agregada (mediante estadística descriptiva y el método de cajas o agrupación de datos por cuartiles) y desagregada (mediante el método de análisis de componentes principales-ACP y el coeficiente de correlación). De esta forma se tiene una visión integral del rol de la gestión del conocimiento en las empresas del sector.

Los resultados del estudio indican que a nivel general, la percepción de los directivos y empresarios de las empresas del sector reconocen la importancia de la gestión del conocimiento para construir una ventaja competitiva, sin embargo, manifiestan que en sus empresas es poco usual la conversión de conocimiento tácito en conocimiento explícito para la visibilidad de la creación de conocimiento, cuando se crea éste. Esto se hace tanto de forma individual y colectiva y por medios formales y el principal componente es la interacción entre personas de la misma área por medios fórmales; situación que es similar para la adquisición de conocimiento del entorno donde el principal componente son los especialistas en las tecnologías de la información.

\section{Industrias Creativas}

El concepto de industria creativa-cultural y la relación de éste con la economía basada en el conocimiento, hace referencia a aquellas industrias que combinan la creación, la producción y la comercialización de contenidos creativos que sean intangibles y de naturaleza cultural (UNESCO, 2013). En este sentido, la globalización ha tenido un profundo impacto en estas industrias y a la vez existe una mayor conciencia de la vital importancia que tienen las industrias culturales en el contexto socioeconómico actual de toda sociedad, tanto por su potencial económico como por servir de elemento para la difusión de la diversidad cultural.

Según Buitrago y Restrepo (2013), las industrias creativas y culturales (ICC), se identifican porque su riqueza está basada en el talento, la propiedad intelectual, la conectividad y la herencia cultural de una región. EI Departamento de Cultura, Medios y Deportes del Reino Unido (DCMS) centra a las industrias creativas como aquellos trabajos que tienen su origen en la creatividad, la habilidad y el talento individual, y tienen el potencial de crear empleos y riqueza a través de la generación y la explotación de la propiedad intelectual. Este concepto, surgió a partir de la identificación de las industrias creativas con una 'nueva economía' impulsada por las tecnologías digitales y estrechamente relacionadas con la economía de la información y el 
conocimiento. De esta manera, la explotación de los derechos de propiedad intelectual (IP) proporciona el enlace crucial entre estos programas, y posicionan a las industrias creativas en la vanguardia de la competitividad económica.

De acuerdo a los estudios realizados por el Gobierno Vasco (2014), las industrias creativas y culturales (ICC) tienen un rol preponderante en la sociedad actual por su relación con la economía del conocimiento, la economía creativa, el valor y la riqueza, las cuales se encuentran en el capital intangible (conocimiento, knowhow, propiedad intelectual, capital relacional, posicionamiento y talento, etc.). Estos estudios son enfáticos en afirmar que las ICC tienen un peso significativo dentro de la estrategia global para la sociedad europea, por su dinamismo y su capacidad para generar empleo, porque promueven la diversidad cultural europea y fomentan la cohesión territorial y porque la creatividad tiene efectos positivos directos e indirectos en la innovación y, por tanto, en el conjunto de sectores económicos.

De igual forma, gracias a los progresos de las tecnologías de la información y las comunicaciones (TIC) y a la apropiación y uso del conocimiento, las industrias culturales y creativas, particularmente las de las economías desarrolladas, cambian sus estructuras, modelos de negocio y la relación entre los creadores y los usuarios, y dan origen a nuevas expresiones culturales. La razón es porque, en las economías desarrolladas suele existir dialogó entre la creación artística y la demanda cultural, entre las dinámicas socioeconómicas y las dinámicas culturales, entre las exigencias políticas y empresariales y las ofertas de actividades culturales (Puig, 2001). Además, según Yzaguirre (2008 y 2014), en los países desarrollados, las organizaciones culturales se preocupan por aprender continuamente de la diversidad, debido a que uno de los fundamentos de la cultura y su riqueza es precisamente la diversidad en sus diferentes manifestaciones.

En este mismo orden de ideas, para las Naciones Unidas (UNCTAD, 2008), las ICC están cimentadas en el uso del conocimiento desde la creatividad y el capital intelectual en los procesos de creación, producción y distribución de bienes y servicios. Para este organismo, el resultado del aporte de estas industrias a la sociedad es visible en productos tangibles y servicios intangibles intelectuales 0 artísticos con contenido creativo, valor económico y objetivos de desarrollo cultural. A este respecto, para el caso colombiano, la generación de valor de las ICC a la economía, ocupa el tercer lugar en número de empleados (1.160 miles de trabajadores) después de México (5.280) y Brasil (1.730). En este sentido, la contribución de esta industria a la economía colombiana es del 3.3\% y a la generación de empleo de 5.8\% (Buitrago y Duque, 2013).

De acuerdo con Kooyman y Hagoort (2010), el concepto de cultura basada en la creatividad reconoce que la combinación de personas con talento, cultura, creatividad, habilidades técnicas y ambientes sociales tienen un impacto importante en el estímulo de la investigación, la optimización de recursos humanos y la inspiración de las personas. Las ICC requieren como input el conocimiento y de labores intensas de las rutinas organizacionales. Según Dervojeda et al. (2013), las Industrias creativas incluyen actividades relacionadas con la creación, producción o distribución de bienes creativos y servicios, así como con la integración de elementos creativos en todos sus procesos y también a los de otros sectores de la sociedad.

\section{Gestión de conocimiento}

Este apartado aborda principalmente los aportes del modelo planteado por McAdam y Reid (2001), el cual toma como insumos los aportes de Baker y Barker (1997), los de la espiral del conocimiento, de Nonaka y Takeuchi (1995), los de la construcción social para el proceso de gestión del conocimiento de Demarest (1997), los de Von krogh et al. (2001) sobre el significado de SECI (socialización, externalización, combinación e interiorización) en la creación de conocimiento y los de Seidler-de Alwis y Hartmann (2008), relacionados con el uso del conocimiento.

Nonaka et al. (1994) identificaron que la importancia del conocimiento tácito en los procesos de creación de conocimiento y el uso del mismo, pueden encontrarse en el centro del proceso de la creación de conocimiento. Según Wagner y Sternberg (1985, citados por Harlow, 2008) definen el conocimiento tácito como el relacionado con el trabajo práctico, conocimiento aprendido informalmente en el trabajo. Estos autores precisan que, sólo una parte del conocimiento tácito, es visible a partir de los conocimientos técnicos. La otra parte del conocimiento tácito es la dimensión cognitiva (Beamer y Varner, 2001, citados por Harlow, 2008) que consiste en las creencias, los valores, las actitudes, los ideales, los mapas mentales y los esquemas que se relacionan con la formación cultural del individuo y del grupo. Esta dimensión cognitiva del conocimiento tácito es la parte más importante, sin embargo, es la más difícil, para lograr la difusión y la creación de conocimiento. De acuerdo a los aportes de Byosiere y Luethge (2008), las teorías de conocimiento identifican y reconocen dos tipos de conocimiento principales: explícito y tácito. El conocimiento explícito se refiere al conocimiento que puede ser traducido al lenguaje formal y sistemático y como tal, es relativamente fácil de reconocer y transferir. El conocimiento tácito, por el contrario, tiene una cualidad personalizada que hace que sea difícil de formalizar, y por lo tanto, está, profundamente arraigado en la acción y el compromiso de las personas en un contexto muy específico. 
En esta misma línea de opinión, para Von Krogh et al. (2001), el conocimiento tácito se asocia con los sentidos, las habilidades motrices, la percepción individual, las experiencias físicas, las reglas prácticas y la intuición. En la realidad empresarial, el reto del buen uso del conocimiento tácito está en las amplias conversaciones constructivas y útiles y en las excelentes relaciones personales basadas en la confianza, la colaboración y la cooperación. En este sentido, la creación de conocimiento a partir del conocimiento tácito y explícito es tanto social como individual. El conocimiento tácito se puede encontrar en las percepciones subjetivas, las intuiciones, los presentimientos y el know-how, y frecuentemente sólo puede ser adquirido a través de la experiencia (Lee Endres et al., 2006). La naturaleza tácita del conocimiento puede derivarse de los procesos técnicos (destrezas personales y know-how) o puede emerger de las cogniciones que son más difíciles de expresar y, por lo tanto, compartir con otros (ideales, valores, modelos mentales). En esencia, el conocimiento tácito sólo existe debido a las personas y su habilidad limitada para entender las experiencias de los otros a través del lenguaje en sí mismo.

Para Senker (1993, citado por Seidler-de Alwis y Hartmann, 2008), el conocimiento tácito puede activarse mediante la generación de nuevos conocimientos científicos (aprender a aprender), mediante la incorporación de nuevos conocimientos en el diseño de un nuevo producto. Se puede activar aún más en el proceso de aprendizaje de nuevos métodos de producción y mejorar la tecnología existente a través de reformas de menor importancia basadas en aprender haciendo y basadas en el aprendizaje de uso. En general, el conocimiento tácito, dentro de la organización puede activarse a partir de las capacidades que poseen las personas, y colocándose como reto las mejoras que podrían hacerse con el aprendizaje acumulado por éstas. Fuera de la organización, se puede obtener a partir de los conocimientos tácitos y las habilidades de otras empresas, a través del reclutamiento de los individuos con una alta educación y experticia. Los conocimientos tácito y explícito son complementarios, lo que significa que ambos tipos de conocimiento son esenciales para la creación de conocimiento.

Al profundizar en la creación de conocimiento, se observa que, Spender (1996), Tsoukas y Mylonopoulos (2004), Zollo y Winter (2002), Peteraf y Barney (2003), coinciden al afirmar que la generación y aplicación de conocimiento deben ser parte de las rutinas organizacionales y obtener como resultados el desarrollo de capacidades dinámicas y el aprendizaje organizacional, los cuales deben ser visibles en el nuevos productos, sistemas y servicios (Teece, 2007). De esta forma, se debe hacer énfasis en que cualquier organización que trate dinámicamente con un ambiente cambiante no solo debería procesar información eficientemente, sino además crear información y conocimiento (Acosta, et al. 2013, Vivas 2013 y Teece, 2007). Sin embargo, se puede decir que la interacción de la organización con su entorno, junto a los medios por los cuales ésta crea y distribuye información y conocimiento, es más importante cuando se trata de la búsqueda para entender de forma activa y dinámicamente a la organización (Teece, 2007 y Nonaka, 1994). Desde esta perspectiva, a partir de los aportes de Von krogh et al. (2001), la creación de conocimiento, exige que las organizaciones promuevan unas interacciones útiles y constructivas que les permitan a las personas ser escuchadas, valoradas y de hablar libremente de sus inquietudes. Estas mismas relaciones propician la formación y autorganización de micro comunidades las cuales son el origen y la fuente de la creación de conocimiento.

Al relacionar los aportes anteriores de creación de conocimiento, con la espiral de conocimiento, propuesta por el modelo de Nonaka et al. (2000), es usual encontrar las formas de conversión de conocimiento, visibles en lo que se denomina el proceso SECI (socialización, externalización, combinación e interiorización). Con la socialización, el conocimiento tácito se comparte a través de una microcomunidad de conocimiento, en la cual cada persona siente compromiso y está motivada de pertenecer a ésta. El beneficio del trabajo común entre los miembros es el desarrollo de la mutua conciencia, el valor por los sentimientos y la experiencia personal, las cuales están ligadas al conocimiento tácito. En síntesis, la socialización es el proceso de conversión de conocimiento tácito de una persona a conocimiento tácito de otra persona a través de la interacción interpersonal y la generación de confianza y la empatía mutua.

La externalización de conocimiento consiste en expresar con palabras prácticas y juicios comunes a través de diálogos que tengan significado. En esta fase del proceso SECl, el lenguaje cobra valor para ayudar a compartir nuevas experiencias como para guiar nuevas nociones. De acuerdo con Nonaka y Takeuchi (1995), un lenguaje figurativo en el que se haga uso de las alegorías, metáforas y analogías es de particular importancia para la creación de los conceptos. Un ejemplo de externalización es la articulación de mejores prácticas y lecciones aprendidas.

La combinación de conocimiento, está encaminada a la presentación de conocimiento explícito existente, debe contar con un ambiente de colaboración, bajo la modalidad de redes en línea o groupware. También se entiende la combinación como el proceso de sistematización de conceptos en un sistema de conocimiento, que ocurre cuando las personas sintetizan diferentes fuentes de conocimiento explícito, por ejemplo, un informe, a través de actividades como las reuniones y las conversaciones de diferente índole y el intercambio de los documentos. Según Nonaka (1994), las personas intercambian y combinan el conocimiento a través de los mecanismos de intercambio como las conversaciones cara a cara. 
La interiorización de conocimiento, se describe como un proceso de incorporar conocimiento explícito en conocimiento tácito. Está estrechamente vinculada a la noción tradicional de aprendizaje y aprender haciendo. Las personas también pueden interiorizar experiencias por la creación y revisión de los documentos. La interiorización igualmente implica, o se logra mediante la difusión de conocimiento explícito a través de una organización. La interiorización es ejemplificada por el aprendizaje que ocurre a partir de la lectura o revisión de material informativo (Alavi y Leidner, 2001).

El proceso SECI, suele coincidir con los aportes de Seidler-de Alwis y Hartmann (2008) quienes afirman, que los prerrequisitos para la evolución del conocimiento tácito y su visibilidad en la innovación, son una cultura abierta a la interacción con otros, en contraposición al aislamiento, y los contactos personales con organizaciones externas o dentro de las organizaciones. Por consiguiente, es deber de la gerencia apoyar y permitir estas interacciones diversas.

Para concluir este apartado, y en complemento a lo ya mencionado, es importante señalar que, en general, la gestión del conocimiento es considerada como un proceso dinámico de creación, adquisición, almacenamiento y transferencia o compartición, y de aplicación de conocimiento, con el fin de contribuir a potenciar la capacidad competitiva de las organizaciones (Simanca et al., 2016, López et al, 2015; López et al. 2014 y Bernal, 2011). En este sentido, esta investigación fue realizada tanto desde el enfoque del modelo SECl como desde el proceso dinámico antes mencionado.

\section{Agregación y desagregación de datos}

De acuerdo con Álvarez et al. (2015), cuando se trata de analizar los datos resultados de la investigación científica, suelen existir dos enfoques: El primero, consiste en analizar información desagregada de conjuntos de los datos, y el segundo, utiliza la información agregada del conjunto de los datos. El análisis de los datos desagregados para explicar el comportamiento individual implica el supuesto de que la relación entre las variables de medición en cuestión es heterogénea entre los individuos o que los subcomponentes de las variables de medición son heterogéneos, y el análisis agregado de los datos para explicar el comportamiento colectivo implica el supuesto de que la relación entre las variables de medición en cuestión es homogénea entre los individuos o que los subcomponentes de las variables de medición son homogéneos (Hsiao, et al. 2005). En este sentido, un análisis de regresión utilizando datos agregados proporcionar conclusiones de los comportamientos de los individuos diferentes de las conclusiones utilizando datos desagregados (Garett, 2003).

A este respecto, Cherry y List (2002), Davis (1997) y Goodfriend (1992) han indicado que, el análisis de los resultados con regresiones utilizando datos agregados suelen diferir de los resultados de regresiones que utilizan los datos desagregados o menos agregados. Afirmación que coincide con la expuesta por Hsiao, et al. (2005) quienes afirman que, la predicción de resultados del análisis de datos agregados, es menos precisa que la predicción basada en el análisis de datos desagregados porque se ignora la heterogeneidad de las puntuaciones de las percepciones individuales o la heterogeneidad de las puntuaciones de cada subcomponente de una macro variable analizada. Para estos autores, es importante evitar que se ignore la heterogeneidad de las puntuaciones en las subunidades y el efecto de la agregación no lineal que es uno de los factores que conduce a la evidencia aparentemente contradictoria entre los resultados del análisis de datos agregados versus desagregados. De otro parte, de acuerdo con Banbura y Modugno (2014), tanto el análisis agregado como el desagregado de los datos del comportamiento de unos sujetos o de una variable es información valiosa que hay que complementar para una mejor comprensión de los comportamientos o variables analizadas.

\section{METODOLOGÍA}

Este estudio, considerado descriptivo, se realizó con la información obtenida de una muestra de 110 directivos de igual número de empresas de la industria cultural y creativa con sede en Bogotá de un total de 150 directivos invitados a participar del estudio. Las empresas en las que laboran los entrevistados corresponden a los siguientes subsectores: desarrolladoras de software e impresión 3D (20\%), publicidad $(16 \%)$ artes escénicas (16\%), video juegos (13\%), música $(11 \%)$, patrimonio y cultura $(5 \%)$, diseño $(5 \%)$, audiovisuales y multimedia (5\%), comunicación y prensa (5\%), otras (4\%). Según el tamaño: grandes $(9 \%)$, medianas $(22 \%)$, pequeñas $(45 \%)$, micro (16\%) y sin precisar tamaño $(8 \%)$. Para la obtención de la información se aplicó un cuestionario con escala Likert, diseñado a partir de los trabajos de McAdam y Reid (2001), de Von krogh et al. (2001) sobre el significado de las formas de conversión del conocimiento (tácito a explícito) SECI para la creación de conocimiento y los aportes de Seidler-de Alwis y Hartmann (2008) relacionados con el uso del conocimiento tácito y los aportes de Blanco (2004), Blanco y Bernal (2009) sobre percepción de la gestión del conocimiento en las empresas colombianas. El cuestionario fue aplicado de forma personalizada, durante el periodo junio a diciembre de 2016, por los autores de la investigación y el apoyo de 
un grupo de estudiantes de seminario de investigación (último semestre de carrera) del programa de Administración de Empresas de la Escuela Internacional de Ciencias Económicas y Administrativas de la Universidad de La Sabana y el profesor Fabio Victorino de la Universidad Santo Tomás a quienes agradecemos por la colaboración. Previa a la aplicación, el cuestionario fue validado mediante muestra piloto y revisión de expertos.

La encuesta evaluó (en escala likert con puntuaciones entre 1 y 7) cuatro aspectos específicos de la gestión del conocimiento: (a) las formas de conversión del conocimiento según el modelo SECI (Socialización - SOC, Externalización - EXT, Combinación - COM, e Interiorización - INT); (b) los medios de la generación de conocimiento (Individual - Indi., Grupal homogéneo - G. Homogéneo., Grupal heterogéneo - G. Hetero., Intergrupal - Inter g., y Mixta - Mixt; Cara a cara formal y planificado - F. Plan., Cara a cara informal y espontáneo -F. Espon, consulta de documentos internos - Doc int., uso de tecnología de información (Tech, Acceso a bancos de datos, foros, mercados - BD); (c) los responsables de la captura y adquisición de conocimiento (especialistas en tecnología de información- Especia., gerencia central -G. Centr., cada persona implicada - Cada pers., gerencia media -Ger. Med. y Nadie y (d) los beneficios de la gestión del conocimiento para la empresa (impacto en la innovación- producto, proceso, marketing y organizacional; en la eficiencia, las ventas, los costos y el aprendizaje).

El análisis de los datos se realizó desde un enfoque integral (datos agregados y desagregados). El agregado mediante estadística descriptiva (estimación de promedios y desviaciones estándar) y agrupación de datos por cuartiles dividiendo el set de datos ordenados en cuatro (4) grupos con la misma cantidad de elementos, tomando los valores límites de cada grupo como el límite de cuartil. El desagregado mediante el método de análisis de componentes principales (ACP) para conocer cómo el cambio de una unidad de una variable afecta en las demás variables relacionadas y mediante el análisis de correlación para indicar qué tanto de la variación de una variable se puede relacionar con la variación de otra - por pares de variables.

\section{RESULTADOS Y DISCUSIÓN}

De manera agregada, los resultados del estudio indican que las empresas del sector practican parcialmente las formas de conversión del conocimiento (socialización, externalización, combinación e interiorización) debido a que el puntaje promedio de las puntuaciones dadas por los entrevistados fue de 5.4 con una desviación estándar de 0.9. Situación que contrasta con lo planteado por Scherrer (2008) y los estudios de la UNCTAD (2008) y del gobierno Vasco (2014) al afirmar que, las industrias creativas y culturales (ICC) tienen un rol preponderante en la sociedad actual por su relación con la economía del conocimiento, la innovación, el valor y la competitividad empresarial.

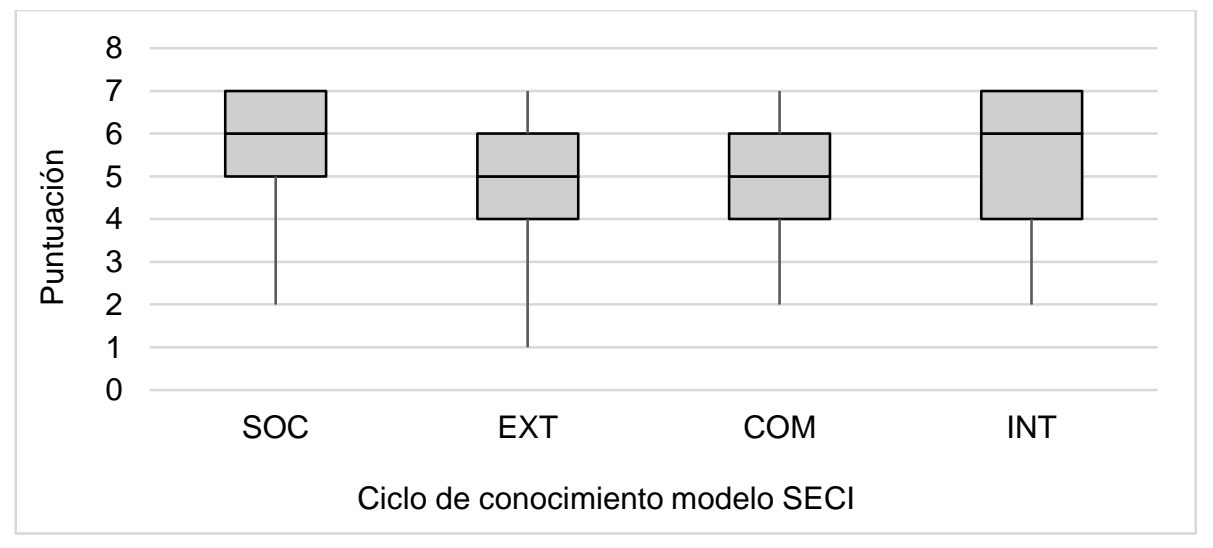

Fig. 1. Puntuaciones de las formas de conversión de conocimiento por cuartiles

No obstante, de la afirmación anterior, el análisis de los datos de forma discriminada (por forma de conversión), la figura 1 muestra que, la socialización es la forma más practicada (el 75\% de las puntuaciones son iguales o superiores a cinco (5) y la externalización y combinación son las menos practicadas (el $50 \%$ de las puntuaciones están entre 4 y 6) y, para la interiorización el 75\% están entre 4 y 7 . En este sentido, con nivel de significancia de $99 \%$, únicamente la socialización fue puntuada con valores superiores a las demás formas de conversión de conocimiento. Esto significa que, en estas empresas se da más importancia al conocimiento tácito que al conocimiento explícito, lo cual es altamente positivo para las empresas como lo evidencia Harlow (2008) al afirmar que, el conocimiento tácito es crucial y necesario en las empresas de hoy.

De otra parte, la tabla 1 muestra que, de acuerdo con los coeficientes de correlación, existe baja correlación positiva entre las puntuaciones de las diferentes formas de conversión del conocimiento del modelo SECI. La 
correlación máxima (0.53) se da entre socialización y externalización y la mínima (0.32) entre externalización e interiorización. Datos estos que confirman la preponderancia del uso del conocimiento tácito sobre el explícito por parte de las personas que laboran en las empresas del sector.

Tabla 1. Coeficientes de correlación entre las formas de conversión del conocimiento

\begin{tabular}{|c|c|c|c|c|}
\hline Conversión de conocimiento & SOC & EXT & COM & INT \\
\hline SOC & 1.00 & 0.39 & 0.37 & 0.43 \\
\hline EXT & & 1.00 & 0.53 & 0.32 \\
\hline COM & & & 1.00 & 0.50 \\
\hline INT & & & & 1.00 \\
\hline
\end{tabular}

En cuanto al análisis de componentes principales de la percepción de los entrevistados referente a las formas de conversión del conocimiento en las empresas, la figura 2 muestra los cuatro componentes de esa percepción (eje horizontal). El primer componente representa el 57\% del total de las variaciones de las puntuaciones en las formas de conversión del conocimiento (eje vertical) y la interiorización es la forma de conversión de conocimiento relevante. Esto quiere decir que, un cambio de una unidad (100\%) en la puntuación de la interiorización explica cambios del $96 \%$ en una unidad de la combinación, del $85 \%$ en la exteriorización y del 68\% en la socialización. El segundo componente representa el 19\% de las variaciones en las puntuaciones, y tanto la interiorización como la externalización son preponderantes, pero el cambio en la interiorización afecta de forma positiva las otras formas, mientras que la exteriorización las afecta de forma negativa. Esto quiere decir que, un cambio en una unidad de la puntuación de la interiorización afecta en un $10 \%$ la socialización, en $-97 \%$ la exteriorización y en -25\% la combinación. El tercer componente representa el $14 \%$ de las variaciones de las puntuaciones y la forma relevante de conversión del conocimiento es la socialización que en los dos primeros perfiles es la menos significante, lo que indica alto grado de independencia de esta forma de conversión de conocimiento respecto de las otras tres formas. El cuarto componente representa el $10 \%$ de las variaciones de las puntuaciones y la forma preponderante de conversión del conocimiento es la combinación. Por la poca variación que representa el perfil 4 la combinación de las formas de conversión del conocimiento en las empresas tiene poco efecto en el total de las percepciones de los encuestados. De estos resultados se deduce que, los cambios en estas empresas suelen ser mediante la noción tradicional de aprendizaje y en particular del aprender haciendo (Alavi y Leidner, 2001).

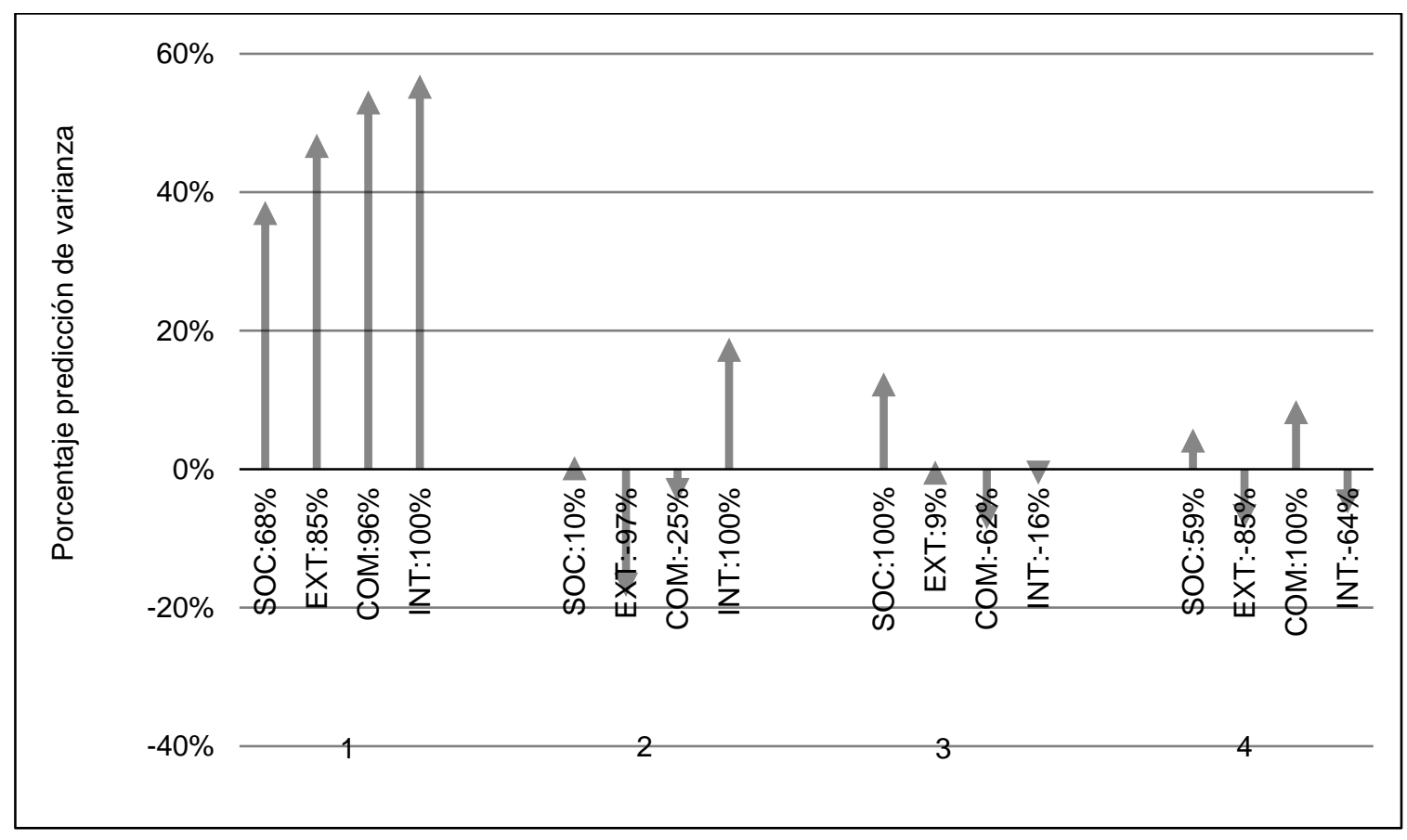

Fig. 2. Componentes principales de las formas de conversión del conocimiento

\section{Medios para la creación de conocimiento}

En lo referente a los medios de creación de conocimiento, los resultados agregados (figura 3) indican que esta creación se hace tanto de forma individual como colectiva (puntajes promedio de 5.5 y 5.2 , respectivamente), rasgo que concuerda con lo que ocurre en las empresas del mismo sector en los países en desarrollo como lo evidencia Kooyman y Hagoort (2010). La creación de conocimiento que se hace 
principalmente a través de medios formales (puntuación 5.6) principalmente mediante el uso de las tecnologías de la información y las comunicaciones, seguida de consulta de fuentes de datos de la empresa y de fuentes externas así como de la asistencia a foros y otras actividades del sector (puntaje promedio 4.8). Medios estos que contrastan con los utilizados por las empresas exitosas en la gestión del conocimiento, las cuales enfatizan en los medios informales - reuniones espontáneas, reuniones alrededor de una taza de café, comunidades de práctica, etc. (Nonaka y Takeuchi, 1995; Teece, 2007 y Vivas, 2013).

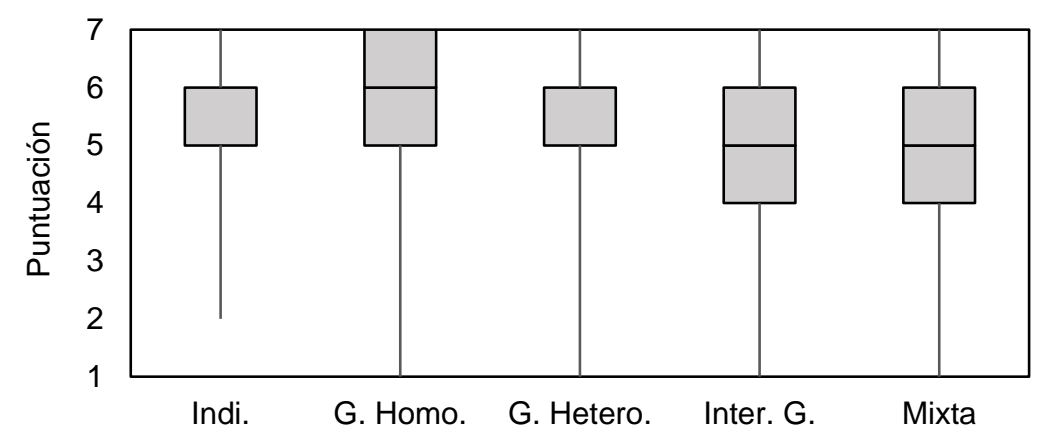

Generación conocimiento individual o colectivo

Fig. 3. Puntuaciones de los medios para creación de conocimiento por cuartiles

De otra parte, los datos de la figura 3 indican que, el medio más usual para generar conocimiento en las empresas es la interacción entre personas de la misma área de actividad (75\% de las puntuaciones es superior a 5) y los medios por los que menos se crea conocimiento es por la interacción entre grupos de diferentes áreas de la empresa (50\% de la puntuaciones está entre 4 y 6). Es de mencionar que, aunque en este sector se suele resaltar tanto el talento individual como el colectivo (Buitrago y Restrepo, 2013), el énfasis en la interacción con las personas de la misma área de trabajo puede ser una debilidad para estas empresas, si se tiene en cuenta la necesidad de una cada vez mayor interacción entre las personas de una empresa con personas de la misma área y de otras áreas y también, con otras personas de otras empresas incluidas las de otros sectores (Seidler-de Alwis y Hartmann 2008).

Los datos de la tabla 2 indican que, existe una correlación positiva entre las puntuaciones de los diferentes medios de creación de conocimiento en las empresas, pero con diferente grado. La correlación máxima se da por la interacción entre grupos de diferentes áreas y grupos interdisciplinares y la mínima ocurre entre personas de la empresa y personas de otras empresas.

Tabla 2. Coeficientes de correlación entre los medios para la creación de conocimiento

\begin{tabular}{|l|r|r|r|r|r|}
\hline $\begin{array}{l}\text { Medios para crear } \\
\text { conocimiento }\end{array}$ & Individual & G. Homo. & \multicolumn{1}{l|}{$\begin{array}{l}\text { Heterogéneo } \\
\text { Inter g. }\end{array}$} & \multicolumn{2}{|l|}{ Mixta } \\
\hline Individual & 1.00 & 0.18 & 0.23 & 0.29 & 0.24 \\
\hline G. Homogéneo. & & 1.00 & 0.39 & 0.39 & 0.13 \\
\hline G. Heterogéneo & & & 1.00 & 0.65 & 0.34 \\
\hline Inter g. & & & & 1.00 & 0.38 \\
\hline Mixta & & & & & 1.00 \\
\hline
\end{tabular}

Los datos de la figura 4 indican que, el primer componente para la creación de conocimiento representa el $50 \%$ del total de las variaciones de las puntuaciones dadas por los entrevistados y el medio preponderante es la interacción intergrupal. Esto significa que, un cambio de una unidad (100\%) en este medio refleja cambios de $40 \%$ en la generación de conocimiento individual, $43 \%$ en la interacción de personas de la misma área, $77 \%$ entre grupos de diferentes áreas y $87 \%$ entre personas de la empresa con personas de otras empresas. En este sentido, la interacción intergrupal es el medio clave para influenciar en las empresas en cambios en los otros medios de creación de conocimiento. El segundo componente representa el $20 \%$, y la actividad generadora de conocimiento es por la interacción entre personas de la empresa y personas de otras empresas. Actividad ésta que se relaciona con los cambios inversos en la generación de conocimiento entre personas de la misma y de diferente área. El tercer componente representa el $13 \%$ y la actividad preponderante es la generación de conocimiento individual, las demás actividades tienen poca importancia, significando que esta forma de generación de conocimiento no se ve afectada por las demás actividades. El cuarto y quinto componente representa poca variación en las puntuaciones y no tiene gran efecto en el total de las percepciones de los encuestados. 


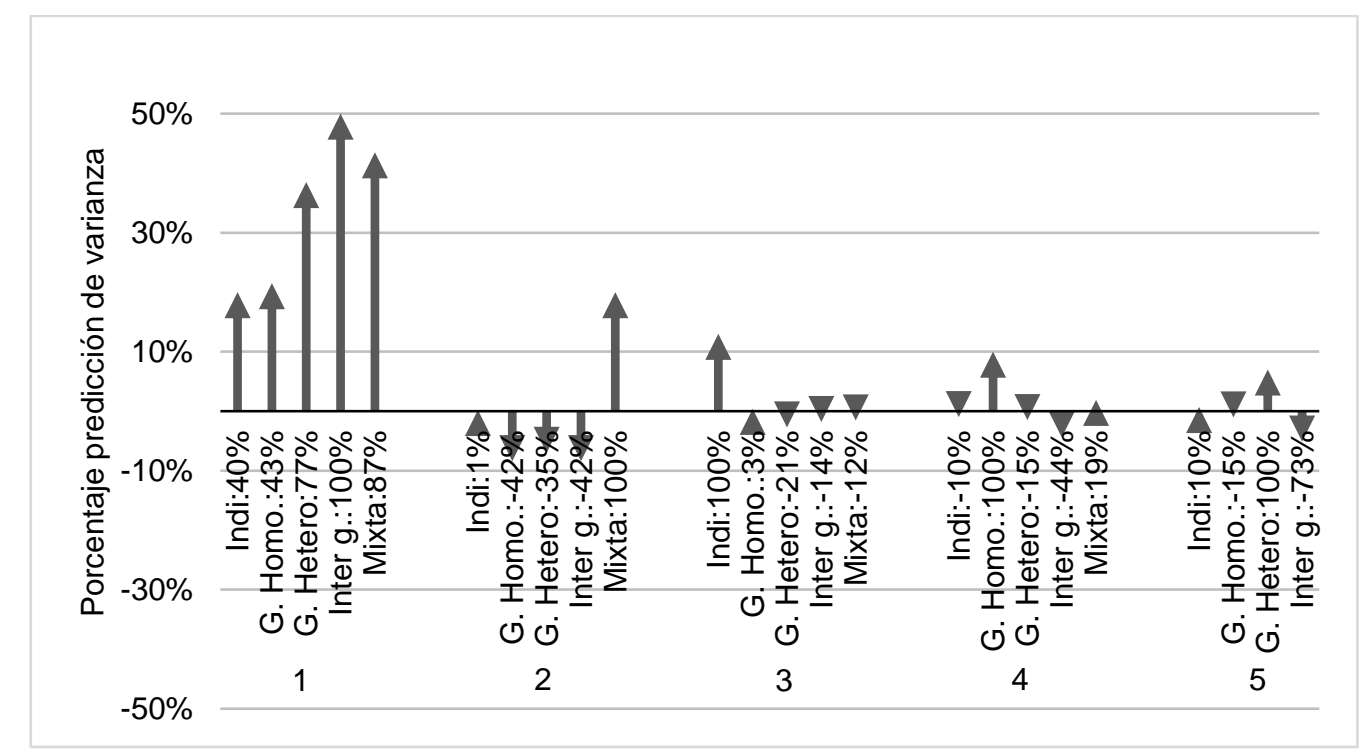

Fig. 4. Componentes principales de los medios para la creación de conocimiento

\section{Responsables de la captura y adquisición de conocimiento}

Los datos agregados de la encuesta indican que en general, en las empresas estudiadas se realiza poca captura y adquisición de conocimiento relevante para el logro de sus objetivos (puntuación promedio 4.4 con desviación estándar de 2.2). Resultados estos, que llaman la atención, si se tiene en cuenta - como ya se mencionó - que la actual es una sociedad en la que conocimiento juega rol dinámico, preponderante y en particular en las empresas de este sector de la creatividad y la cultura (Gobierno Vasco, 2014 y Unctad, 2008).

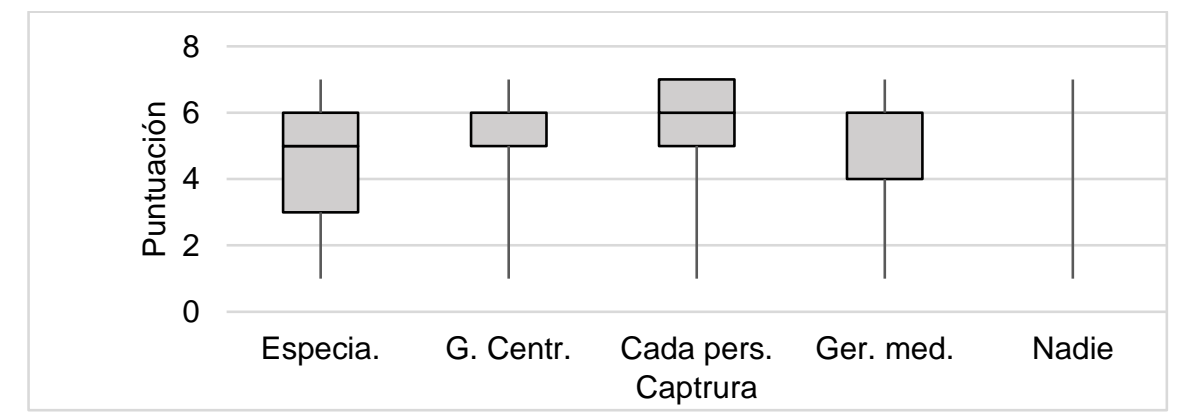

Fig. 5. Puntuaciones por cuartiles de captura y adquisición de conocimiento

Cuando se realiza esta actividad se hace principalmente por la iniciativa que cada persona tiene para el desempeño de sus actividades en la empresa (75\% de las personas puntuaron entre 5 y 7 ), seguida de la responsabilidad de los directivos (50\% puntúa entre 5 y 6 ) y muy poco los responsables o especialistas en tecnologías de la información (50\% puntúa entre 3 y 6 ). A este respecto es de señalar que, en las empresas es muy importante la iniciativa personal para la identificación y la captura de conocimiento relevante para el desempeño laboral y el logro de los objetivos organizacionales, pero también, es importante la existencia de una cultura organizacional liderada por la gerencia que valore y estimule estos comportamientos tanto individuales como colectivos (Nonaka y Takeuchi, 1995; Teece, 2007 y Bernal, 2011). En el proceso de adquisición de conocimiento, los datos de la tabla 3 , muestran que solo existe relación de dependencia entre las actividades de la gerencia central y las de la gerencia media.

Tabla 3. Correlación entre los responsables de la captura y adquisición de conocimiento

\begin{tabular}{|l|r|r|r|r|r|}
\hline Resp. Captura / Cto & Especia. & \multicolumn{1}{|l|}{ G. Centr. } & \multicolumn{1}{l|}{ Cada pers. } & Ger. med. & Nadie \\
\hline Especia. & 1.00 & 0.28 & 0.14 & 0.28 & 0.05 \\
\hline G. Centr. & & 1.00 & 0.17 & 0.55 & 0.10 \\
\hline Cada pers. & & & 1.00 & 0.22 & 0.02 \\
\hline Ger. med. & & & & 1.00 & 0.07 \\
\hline Nadie & & & & & 1.00 \\
\hline
\end{tabular}


La figura 6 muestra los cinco componentes principales de la captura y adquisición del conocimiento. El primer componente representa el $42 \%$ del total de las variaciones de las puntuaciones dadas por los participantes a este aspecto y la variable relevante son los especialistas en las tecnologías de la información; el segundo componente representa el $22 \%$ y al variable relevante también son los especialistas en tecnología; el tercer componente el $15 \%$ y el cuarto el $15 \%$ y el quinto $6 \%$. Indicando con ello que entre el primero y el segundo componente representa el $64 \%$ del total de las variaciones de las puntuaciones dadas por los participantes a este aspecto. Estos resultados son coherentes con el enfoque tecnocrático de la gestión del conocimiento que enfatiza las tecnologías de la información y las comunicaciones como un medio clave para ese propósito cuando se trata de compartir el conocimiento explícito (Bernal 2011 y López, 2015).

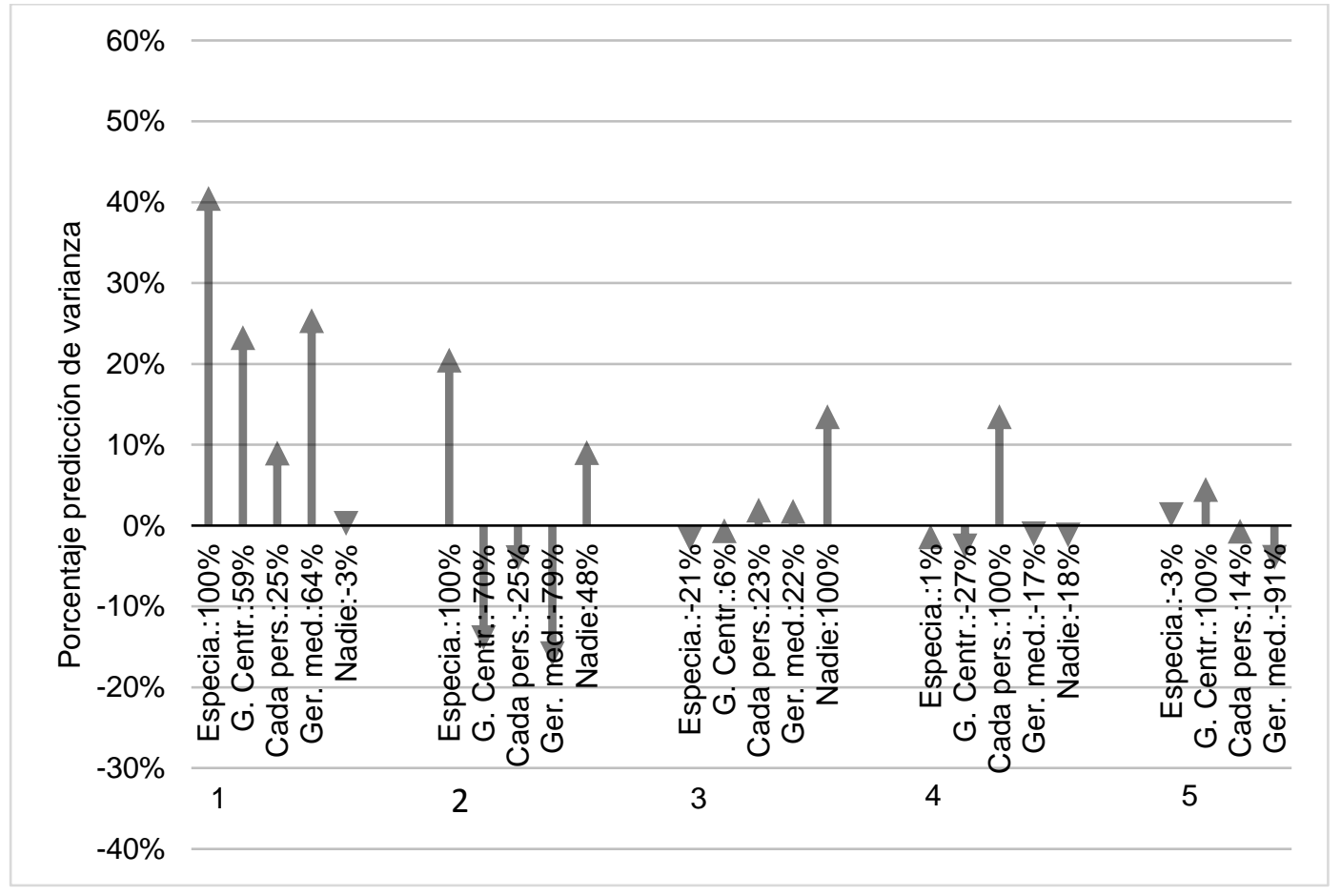

Fig. 6. Componentes principales de la captura y adquisición del conocimiento

\section{Formas de compartir conocimiento}

Los resultados de la encuesta indican que, en general en las empresas estudiadas es poco usual compartir conocimiento relevante para el logro de sus objetivos (puntuación promedio 4.4 con desviación estándar de 2.2). Situación que es similar a otras antes mencionadas en este mismo estudio contrasta con la cultura de las organizaciones en la economía del conocimiento, donde compartir conocimiento es una de sus estrategias claves para potenciar su capacidad competitiva (Nonaka y Takeuchi, 1995, McAdam y Reid, 2001, Blanco y Bernal, 2009 y Bernal 2011).

De otra parte, la figura 7 , indica que, cuando en estas empresas se comparte conocimiento, se hace de manera formal (el $75 \%$ de las puntuaciones están entre 5 y 7 ).

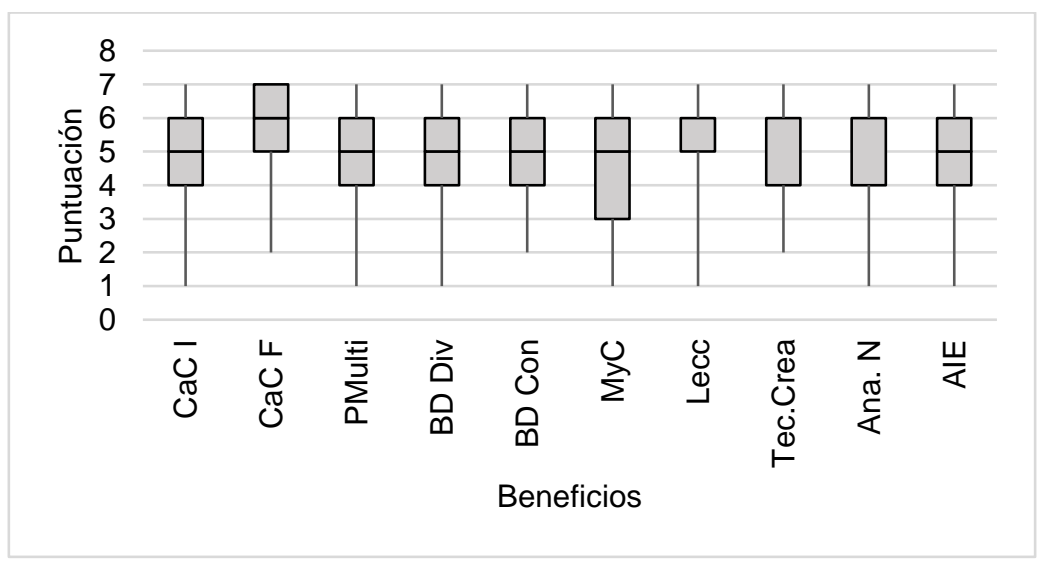

Fig. 7. Puntuaciones en cuartiles de los medios de compartir conocimiento 
Las mayores correlaciones en cuanto a compartir conocimiento en las empresas se dan entre los procesos de aprendizaje y entrenamiento para el cargo y las necesidades de capacitación (0.7); seguida de la relación entre el análisis de las necesidades de capacitación y entrenamiento y el uso de técnicas creativas (0.63) el uso de bases de datos de fuentes relacionadas con las actividades de las empresas (0.58), el uso de lecciones aprendidas (0.56) y el uso de fuentes de información diversas (0.54).

Tabla 4. Coeficientes de correlación medios para compartir conocimiento

\begin{tabular}{|c|c|c|c|c|c|c|c|c|c|c|}
\hline Medios & CaCl & CaC F & PMulti & BD Div & $\begin{array}{c}\text { BD } \\
\text { Con }\end{array}$ & MyC & Lecc & Tec.Crea & Ana. N & AIE \\
\hline & & & & & & & & & & \\
\hline CaC4 I & 1.00 & 0.23 & 0.19 & 0.01 & 0.02 & 0.07 & 0.20 & 0.32 & 0.20 & 0.20 \\
\hline CaC F & & 1.00 & 0.47 & 0.40 & 0.46 & 0.17 & 0.43 & 0.39 & 0.33 & 0.17 \\
\hline PMulti & & & 1.00 & 0.55 & 0.00 & 0.37 & 0.38 & 0.34 & 0.43 & 0.34 \\
\hline BD Div & & & & 1.00 & 0.73 & 0.40 & 0.40 & 0.43 & 0.54 & 0.32 \\
\hline BD Con & & & & & 1.00 & 0.45 & 0.45 & 0.46 & 0.58 & 0.45 \\
\hline MyC & & & & & & 1.00 & 0.37 & 0.35 & 0.54 & 0.45 \\
\hline Lecc & & & & & & & 1.00 & 0.55 & 0.56 & 0.22 \\
\hline Tec.Crea & & & & & & & & 1.00 & 0.63 & 0.45 \\
\hline Ana. N & & & & & & & & & 1.00 & 0.70 \\
\hline AlE & & & & & & & & & & 1.00 \\
\hline
\end{tabular}

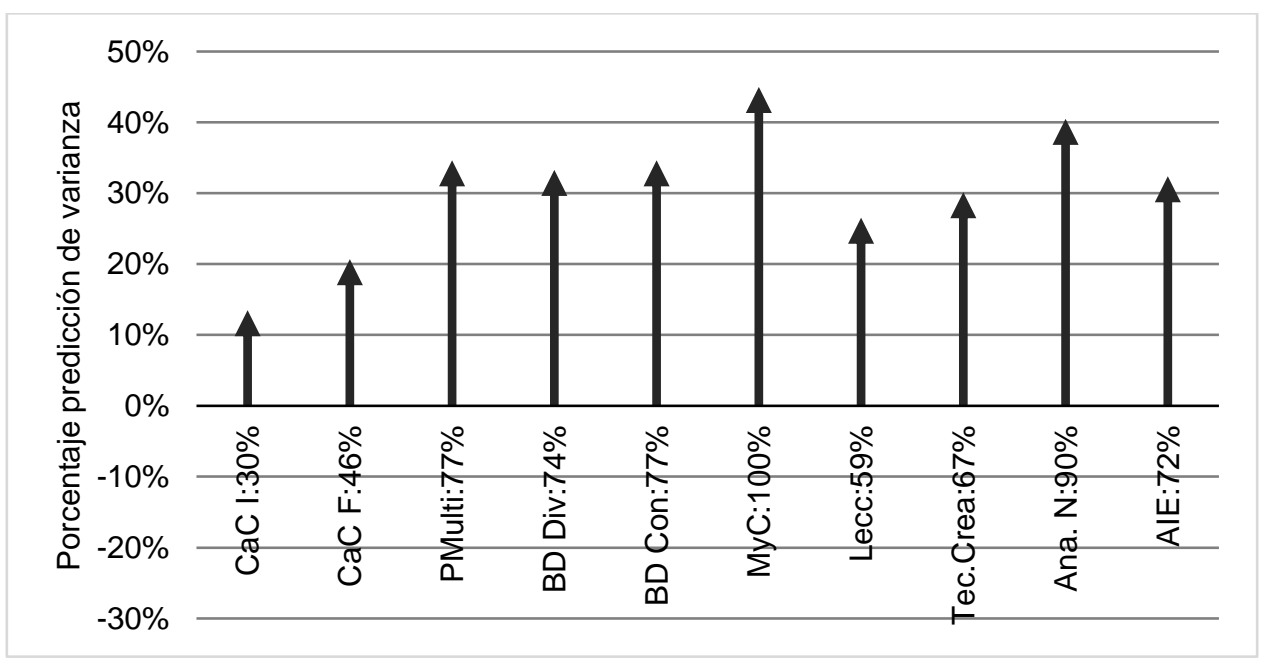

Fig. 8. Componentes principales de los medios de compartir conocimiento. Primer componente principal

De acuerdo con los datos de figura 8, el componente principal de los medios para compartir el conocimiento en las empresas representa el $45 \%$ del total de las variaciones de las puntuaciones dadas por los participantes y está representado por el mentoring y el coaching. Esto significa que aunque este medio es el menos usado, es el que más impacto tiene en los demás medios de compartir conocimiento. De otra parte, los demás (9) componentes representan un muy bajo impacto en las variaciones de las puntuaciones del uso de los diferentes medios de compartir conocimiento.

\section{Beneficios de la gestión de conocimiento}

Para efectos de este estudio, los beneficios evaluados de la gestión del conocimiento para las empresas del sector, y que se representan en el eje horizontal de la figura 9 fueron: $10 \mathrm{a}=$ mejora en los bienes o servicios de la empresa, $10 \mathrm{~b}=$ oferta de nuevos bienes o servicios, $10 \mathrm{c}=$ mejoras en los procesos de producción de los bienes o servicios, $10 \mathrm{~d}=$ Cambios en la forma de administrar de la empresa, $10 \mathrm{e}=$ cambios en las actividades del marketing, $21 \mathrm{a}=$ mejora en le eficiencia, $21 \mathrm{~b}=$ incremento en los ingresos financieros, $21 \mathrm{c}=$ diversificación de bienes o servicios, $21 \mathrm{~d}=$ reducción de costos de operación, $21 \mathrm{e}=$ mejor aprendizaje organizacional, $21 \mathrm{f}=$ reducción de tiempos de producción de bienes o servicios y $21 \mathrm{~g}=$ mejor servicio al usuario o cliente. 
Los resultados de esta evaluación indican que, los encuestados perciben que la gestión sistemática del conocimiento tiene impacto positivo en diferentes actividades y en los resultados de las empresas (puntuación promedio 5.8 con desviación estándar de 1.0). Sin embargo, de acuerdo con la figura 9, los mayores impactos se reflejan en las mejoras y los cambios en los procesos de actividad de las empresas y en la mejora de la eficiencia empresarial (75\% de las puntuaciones están entre 6 y 7 ). Los menores impactos se perciben en las mejoras o cambios en las actividades de marketing (50\% de las puntuaciones están entre 5 y 6$)$. Aquí llama la atención, la percepción del poco y casi nulo impacto de la gestión del conocimiento en la innovación de las empresas, particularmente la innovación de productos (bienes/servicios), esto porque, en los resultados de las investigaciones en empresas en países desarrollados es usual encontrar relación positiva entre gestión del conocimiento e innovación (Nonaka y Takeuchi, 1995 y Blanco, 2004), pero la percepción coincide con los resultados de estudios realizados en Colombia sobre gestión del conocimiento de las medianas y grandes empresas (Blanco y Bernal 2009 y López et al. 2015).

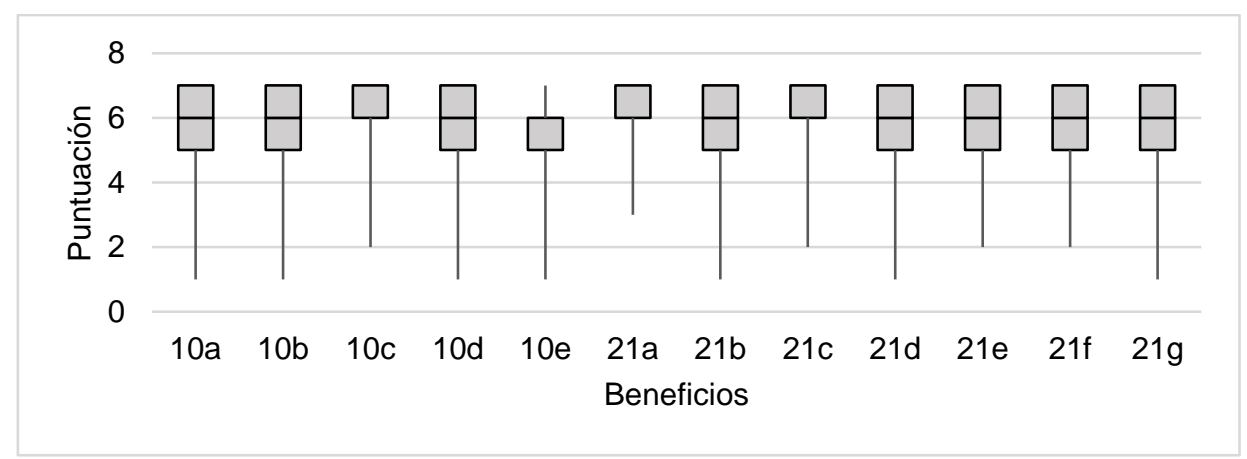

Fig. 9. Puntuaciones en cuartiles de los beneficios de la gestión del conocimiento

Para finalizar, cabe señalar que, el bajo impacto de la gestión del conocimiento en la generación de nuevos bienes o servicios, en la diversidad de oferta de éstos y en el cambio de los estilos administrativos de las empresas, obedece a la poca importancia que en la actualidad, en Colombia, se da a la gestión del conocimiento, no solo en el campo de la cultura y la creatividad, sino en general en los diversos sectores de la economía (Bernal et al, 2014) y al estilo tradicional de administrar las organizaciones basado en la autoridad y la jerarquía. Situación que contrasta con la importancia que en las empresas del sector, de los países desarrollados, se da a la gestión del conocimiento como estrategia de competitividad y el estilo flexible de administrar las organizaciones que permite la autonomía en la toma de decisiones y el diálogo entre las empresas del sector, los usuarios y los responsables de las políticas públicas en lo referente al estímulo de la cultura y la creatividad (Puig, 2001).

\section{CONCLUSIONES}

En general, según el análisis agregado de los datos, los directivos y empresarios de las empresas culturales y creativas entrevistados reconocen la importancia de la gestión del conocimiento para construir ventaja competitiva en esas empresas. Sin embargo, manifiestan que en sus empresas, es poco usual la conversión de conocimiento tácito en conocimiento explícito. El conocimiento que en ellas se crea se hace tanto de forma individual como colectiva principalmente por medios formales y particularmente por la interacción entre personas de la misma área. El conocimiento del entorno se adquiere a través de especialistas en las tecnologías de la información.

De otra parte, el análisis desagregado de los datos (componentes principales) permitió identificar que, en esas empresas el medio de conversión de conocimiento que más impacta es la interiorización del conocimiento y no la socialización que es sugerida por los expertos para una eficaz gestión del conocimiento; la forma de mayor impacto en la creación de conocimiento es la interacción intergrupal y que no es la que se practica en esas empresas.

Los resultados de este estudio han permitido un acercamiento al conocimiento de la realidad de las industrias culturales y creativas en lo referente a la gestión del conocimiento y sirve de referente para que académicos y directivos empresariales y de organismos gubernamentales interesados y con responsabilidades en el sector reflexionen y originen acciones, ojala conjuntas, que permitan el diseño de estrategias soportadas en la gestión del conocimiento que contribuyan a generar valor agregado distintivo y sostenible para las empresas de esta industria y sus diferentes grupos de interés. Esto es muy importante, si se tiene en cuenta que éste es un sector con gran impacto en las economías nacionales, especialmente en las de los denominados países desarrollados y que requiere de un gran impulso en economías emergentes, por el poco valor que en estas economías se le da a la actividad cultural. 


\section{REFERENCIAS}

Acosta, J., Longo-Somoza, M. y Fischer, A. Capacidades dinámicas y gestión del conocimiento en nuevas empresas de base tecnológica, Cuadernos de Administration ser. Organ: 26 (47), 35-62, (2013).

Alavi, M y Leidner, D. Knowledge Management and Knowledge Management Systems: Conceptual Foundations and Research Issues, Management Information Systems Research Center, University of Minnesota: 25(1), 107-136, (2001).

Alvarez, R. Camacho, M. y Perez-Quiroz, R. Aggregate versus disaggregate information in dynamic factor models. En: http://www.um.es/econometria/Maximo/articulos/SSvLS.pdf (2015). Consultado el 23 de marzo de 2017.

Baker,M y Barker,M. Human Capital, Journal of Knowledge Management, 1(1), (1997).

Banbura, M., and Modugno, M. Maximum likelihood estimation of factor models on datasets with arbitrary pattern of missing data. Journal of Applied Econometrics: 29, 133-160 (2014).

Bernal, C, Frost, S. y Sierra, H. Importancia de la gerencia del conocimiento: contrastes entre la teoría y la evidencia empírica. Estudios gerenciales: 30(130), 65-72 (2014).

Bernal, C. A, Modelo de gerencia del conocimiento: visión integral, Cuadernos de Administración: 27(46), 55-66 (2011).

Blanco, C.E y Bernal, C. Percepciones sobre la gestión del conocimiento por una muestra de directivos de grandes y medianas empresas en Bogotá, Revista Cultura, Tecnología y Patrimonio, Centro Universitario de Los Valles, Guadalajara, México, (2009).

Buitrago y Duque. La Economía Naranja: Una oportunidad infinita, Banco Interamericano de Desarrollo (2013).

Byosiere, $\mathrm{P}$ y Luethge, D.J. Knowledge domains and knowledge conversion: an empirical investigation, Journal of Knowledge Management: 12(2), 67-78, (2008).

Caggiano, G., Kapetanios, G., and Labhard, V. Are more data always better for factor analysis? Results for the Euro area, the six largest Euro area countries and the UK, Journal of Forecasting: 30, 736-752 (2011).

Cherry, T. y J. List, Aggregation bias in the economic model of crime, Economics Letters: 75, 81-86, (2002).

DCMS. Creative Industries Mapping Document. London: DCMS, (1998).

Demarest. Understanding Knowledge Management. Long Range Planning: 30, 374-384 (1997).

Dervojeda,K; Nagtegaal,F; Lengton,M y Peyoush Datta. Creative industries Analysis of industry-specific framework conditions relevant for the development of world-class clusters. Enterprise and Industry Directorate-General of the European Commission, European Union, (2013).

Davis, G. Product aggregation bias as a specification error in demand systems, American Journal of Agricultural Economics: 79, 100-109, (1997).

Florida, R. The Rise of the Creative Class, Basic Books, New York, (2002).

Garrett, T. A ggregated versus disaggregated data in regression analysis: implications for inference. Economics Letters: 81, 61-65 (2003).

Gobierno Vasco. Las industrias culturales y creativas: Debate Teórico desde la Perspectiva Europea. Departamento de educación, política lingüística y cultura, Vitoria-Gasteiz (2014).

Goodfriend, M. Information-aggregation bias, American Economic Review: 82, 508-519 (1992).

Harlow, H. The effect of tacit knowledge on firm performance, Journal of Knowledge Management: 12(1), 148-163, (2008).

Hsiao, C., Shen, Y., and Fujiki, H. Aggregate vs. Disaggregate data analysis-aparadox in the estimat ion of a money demand function of japan under the low interest rate polic, Journal of applied econometrics: 20, 579-601 (2005).

Kooyman, R y Hagoort, G. The Entrepreneurial Dimension of the Cultural and Creative Industries, Utrecht School of the Arts, The Faculty of Art y Economics and the Faculty of Art, Media y Technology. The Netherlands, (2010).

Lee Endres, M; Endres, S, Chowdhury, Sanjib; Alam, Intakhab. Tacit Knowledge Sharing, Self-Efficacy Theory, And Application To The Open Source Community, Journal of Knowledge Management: 11(3), 92 - 103 (2006).

López, M., A. Hernández y C. Marulanda, Procesos y prácticas de gestión del conocimiento en cadenas productivas de Colombia, Información Tecnológica: 25(3), 125-134 (2014).

López, D., Marulanda, C. y López, M. Métricas de Valoración de la Gestión del Conocimiento para las Pequeñas y Medianas Empresas del Sector Tecnologías de Información en el Triángulo del Café en Colombia., Información tecnológica: 26(3), 173-183 (2015).

McAdam, R y Reid, R. SME and Large organization of Knowledge management: comparisons and Contrasts. Journal of Knowledge Management, 5(3), 231-241 (2001).

Nonaka, I y Takeuchi, H. The Knowledge-creating company: How Japanese companies create the -dynamics of innovation, Oxford University Press, Nueva York, (1995).

Nonaka, I., Byosiere, P., Borucki, C. y Konno, N. Organizational knowledge creation theory: a First comprehensive test, International Business Review: 3(4), 337-51 (1994). 
Nonaka, I., Toyama, R. y Konno, N. SECl, ba and leadership: a unified model of dynamic knowledge creation, Long Range Planning: 33, 4-34 (2000).

Peteraf, M.A. y Barney, J. Unravelling the resource-based tangle, Managerial and Decision Economics: 24(4), 309-24 (2003).

Puig, T. Se acabó la diversion: Ideas y gestión para la cultura que crea y sostiene ciudadanía, Libros del Rojas, Buenos Aires, (2001).

Scherrer, W. Cultural Industries, Knowledge Transmission, and Regional Innovation Activity:Performing Arts in Salzburg, Prepared for the International Conference of the Regional Studies Association] (2008).

Seidler-de Alwis y Evi Hartmann. The use of tacit knowledge within innovative companies: knowledge management in innovative enterprises, Journal of Knowledge Management: 12(1), 133-147 (2008).

Simanca, M. Montoya, L. y Bernal, C. Gestión del conocimiento en la cadena láctea Colombia, Revista Información tecnológica: 27(3), 93-106 (2016).

Spender, J.-C. Making knowledge the basis of a dynamic theory of the firm, Strategic Management Journal: 17, 45-63 (1996).

Teece, D. Explicating dynamic capabilities: The nature and microfoundations of (sustainable) enterprise performance, Strategic Management Journal, 28, 1319-1350 (2007).

Tsoukas, H. y Mylonopoulos, N. Introduction: Knowledge construction and creation in organizations, British Journal of Management: 15(1), 1-8 (2004).

UNCTAD. Creative economy. Report 2008, UNDP-UNCTAD, Geneva-New York. - (2010): Creative economy. Report 2010, UNDP-UNCTAD, Geneva-New York (2008).

UNESCO. Comprender las Industrias Creativas: Las estadísticas como apoyo a las políticas públicas (2013).

Von Krogh, G., Ichijo, K. y Nonaka, I. Facilitar la Creación de Conocimiento, Oxford University Press, Oxford (2001).

Yzaguirre, F. Industrias culturales y clúster de excelencia urbana. En: Cultura, territorio, economía y ciudad: textos de las primeras jornadas sobre ciudades creativas organizadas por la Fundación Kreanta en Sitges (Barcelona) en noviembre de 2008. Félix Manito (ed.). Fundación Kreanta, Barcelona. 111-125 (2009)

Yzaguirre, F. La gestión de la calidad, el conocimiento y el talento en las organizaciones culturales, Manual AtalayaUniversidad de Cádiz, Cádiz, (2014).

Zollo, M. y Winter, S.G. Deliberate learning and the evolution of dynamic capabilities, Organization Science: 13(3), 339-51 (2002). 